\title{
EcoÁgua: M-learning e gamification como estratégias de suporte ao desenvolvimento do consumo sustentável de água
}

\author{
Andreza Priscila de L. Ferreira ${ }^{1}$, Juliana Basto Diniz ${ }^{2}$, José E. G. da Silva Júnior ${ }^{3}$ \\ 123 Unidade Acadêmica de Educação a Distância e Tecnologia- Universidade Federal \\ Rural de Pernambuco (UFRPE) \\ Rua Dom Manoel de Medeiros, s/n - Dois Irmãos - CEP: 52171-900 - Recife - PE - \\ Brazil \\ andreza85ufrpe@gmail.com, julianabdiniz@gmail.com,
}

\begin{abstract}
Discussions on environmental issues have started years ago. In fact, recently, this issue has been discussed a lot because of the perception of the damage caused to the environment regarding the resulting pressures of social development on natural resources. Different sectors of society have adopted sustainable practices as a means to contribute to minimize such damage. This paper proposes the development of information actions on environmental issues and use of an application for mobile devices, EcoAgua, to encourage the responsible use of water. Thus, it is expected with the ongoing research, to provoke a critical reflection in relation to environmental issues and to contribute to the development of responsible water.
\end{abstract}

Resumo. As discussões sobre questões ambientais já existem há muito tempo. De fato, recentemente, esta questão foi muito discutida por causa da percepção dos danos causados ao meio ambiente resultantes do desenvolvimento social. Diferentes setores da sociedade adotaram práticas sustentáveis como meio de contribuir para minimizar tais danos. Este trabalho propõe o desenvolvimento de ações de informação sobre questões ambientais e uso de um aplicativo para dispositivos móveis, EcoÁgua, para incentivar o uso responsável da água. Assim, espera-se com a investigação em curso, provocar uma reflexão crítica em relação às questões ambientais e contribuir para o consumo responsável da água.

\section{Introdução}

Não é de hoje que a educação ambiental vem sendo tema de discussões em todo mundo. Estudos apontam que tais discussões, bem como movimentos e eventos internacionais iniciaram entre as décadas de 70 e 90 [Dias 2003]. A realidade nos mostra cidades cada vez mais populosas, com um crescente número de construções civis e para atender as necessidades desta população há um aumento na pressão sobre o meio ambiente resultando na degradação ambiental [Goulart \& Callisto 2003]. Através da educação ambiental busca-se compreender a relação entre homem-natureza, estudando as mudanças ambientais na sociedade e contribuindo com estudos que visem mudar a atual realidade de degradação ambiental, por meio dela tem-se o objetivo de formar cidadãos preocupados com a sustentabilidade usando racionalmente os recursos naturais. Neste cenário destaca-se o papel da educação, principalmente o que diz 
respeito às Universidades por serem produtoras do conhecimento científico contribuindo e influenciando diretamente e indiretamente na formação e desenvolvimento da sociedade. Tal influência refletirá nos pensamentos e posicionamentos dos futuros profissionais graduados destas instituições. Estes, a partir de uma visão e consciência ambiental desenvolvida, atuarão em favor do meio ambiente com ações de influência na degradação ambiental [Marcomin \& Silva, 2009].

Porém verifica-se por meio de estudos na área que não há um dinamismo na inserção das preocupações ambientais nas Universidades, o que não ocorre apenas no Brasil. As ações sustentáveis acontecem de maneira pontual com iniciativas basicamente de docentes e pesquisadores. Tais ações na maioria das vezes não inclui a integração do ensino, pesquisa e extensão [Marcomin \& Silva, 2009].

Nesse cenário insere-se a Unidade Acadêmica de Educação a Distância da Universidade Federal Rural de Pernambuco. Considera-se neste sentido, que a ausência ou as poucas práticas sustentáveis na Unidade deve-se não só a complexidade dessa integração referida por tais estudos, como também ao pouco conhecimento teórico e/ou prático sobre educação ambiental e sustentabilidade. Isso reflete não somente no desconhecimento de quais ações podem ser implantadas, como também em pouco incentivo ao desenvolvimento de ações sustentáveis nesse meio.

Nesse sentido, este artigo apresenta um aplicativo para dispositivos móveis que, associado a algumas ações educativas, tem como objetivo incentivar a mudança na cultura de consumo dos recursos naturais, sobretudo o consumo consciente de água, um recurso natural que vem se tornando escasso nos últimos anos.

O artigo está dividido em cinco seções. A segunda seção apresenta o referencial teórico através de uma discussão no contexto da educação ambiental e do consumo hídrico consciente. Ainda retrata a tecnologia a favor da educação ambiental, sobretudo fazendo uso de aspectos de aprendizagem móvel e gamificação. A terceira seção apresenta a metodologia utilizada no desenvolvimento da pesquisa enquanto a seção 4 apresenta os trabalhos relacionados. Já a seção 5 apresenta o aplicativo EcoÁgua e a última seção apresenta as considerações finais e discussões.

\section{Referencial Teórico}

As causas da degradação ambiental são as mais diversas e decorrentes das mais variadas pressões sobre os recursos naturais. Tais pressões são provocadas pelo contingente populacional que atua como agente modificador da natureza. Considera-se inclusive que em todo o planeta, praticamente não existe um ecossistema que não tenha sofrido influência direta e/ou indireta do homem [Carvalho, et. al., 2009] [Goulart \& Callisto 2003].

Conforme documento da UNESCO [2015], a água está no centro do desenvolvimento sustentável e a gama de serviços que esse recurso provê contribui para a redução da pobreza, o crescimento econômico e para a sustentabilidade ambiental. Atualmente, a sociedade vivencia as consequências do uso insustentável dos recursos naturais. A água doce, por exemplo, já evidencia um quadro de escassez para atender as necessidades humanas. Assim, torna-se indispensável um equilíbrio entre demanda e oferta caso contrário, o mundo enfrentará um déficit global de água cada vez mais grave [Giacomin \& Ohnuma 2012]. Fatores como o crescimento da população, a urbanização, 
as políticas de segurança alimentar e energética, processos macroeconômicos, tais como a globalização do comércio, as mudanças na dieta e o aumento do consumo influenciam no consumo hídrico [UNESCO 2015]. As consequências são perceptíveis em diversas regiões do mundo, rios que atualmente se encontram secos, lagos que estão desaparecendo, entre outras. Diante do cenário de consumo mundial, há uma necessidade em reduzir a demanda de água em escala global [Giacomin \& Ohnuma 2012].

O conceito de sustentabilidade, apesar de bastante difundido, é pouco compreendido e posto em prática. Além disso, há compreensões equivocadas sobre a verdadeira essência deste termo. A sociedade tem ciência de sua importância, mas na maioria das vezes não pratica hábitos sustentáveis em seu cotidiano. Nesse contexto, desenvolver a comunicação sobre esta problemática é de extrema importância, além de informar sobre, tal ação pode incentivar atitudes futuras com vistas à promoção de alguma mudança [Araújo 2015].

Considerando os avanços tecnológicos experimentado nos últimos 20 anos, é evidente que a educação ganha cada vez mais espaço, estando aliada a tecnologia que potencializa o processo de ensino aprendizagem. Sendo assim, por que não utilizar a tecnologia a favor da educação ambiental, uma vez que a mesma está inserida diariamente nas diversas aplicações cotidianas das pessoas?

Nesse sentido destacam-se os jogos eletrônicos digitais que estimulam o raciocínio por exigir estratégias para resolver problemas e testes. Também merece destaque o mobile learning (m-learning) que concilia as características do e-learning com a computação móvel possibilitando mobilidade ao processo de ensinoaprendizagem assim, proporciona novas experiências e um novo panorama em relação ao uso dos Ambientes virtuais de aprendizagem aliados a mobilidade [Mühlbeier et. al. 2012].

Uma nova perspectiva que trata essa questão e incorpora mecânicas de jogos a softwares de diversas áreas vem ganhando força, sendo conhecida como Gamificação, do inglês Gamification [França \& Reategui, 2013. Nesse sentido, a proposta de gamificação se apresenta como uma alternativa no sentido de incluir elementos de jogos em situações que não são necessariamente remetidas como jogos [Viega 2015].

A gamificação ocorre quando elementos de design de jogo (por exemplo, pontos, quadros de líderes, e crachás) são usados em contextos diferentes para promover o engajamento do usuário. Para Deterding e colegas, o uso de elementos de jogos em um contexto de não-jogos tem se popularizado bastante nos últimos anos [Deterding et al, 2011]. A gamificação pode tomar várias formas incluindo o uso de narrativas para mudar o contexto em torno de uma atividade típica

O aprendizado móvel acontece quando a interação é realizada por meio de dispositivos móveis como smartphones, notebooks, tablets, entre outros. De acordo com O'Malley e colegas [O'Malley et al, 2003], o mobile learning pode ser definido como qualquer tipo de ensino que ocorre quando um indivíduo não se encontra num local predeterminado ou fixo, ou quando o indivíduo tem oportunidades de aprendizagem através de dispositivos móveis, podendo assim associar os conceitos tecnológicos e de mobilidade. 
Para Traxler [2007], alguns defensores da aprendizagem móvel tentam defini-la e contextualizá-la em termos de dispositivos e tecnologias; outros, definem e conceitualizam em termos de mobilidade dos aprendentes e da mobilidade da aprendizagem relacionada à experiência dos aprendentes com a aprendizagem através de dispositivos móveis.

Atualmente os dispositivos móveis não são vistos apenas como sinônimo de diversão, após a inserção no mercado dos smartphones e o uso do sistema operacional Android, cresceu o número de aplicações desses aparelhos que antes eram usados principalmente ou exclusivamente para fazer ligações. Hoje, além dessa função, eles também são utilizados para entretenimento, negócios, ensino, aprendizagem, jogos entre outras [Kaur \& Bhullar 2013].

As tecnologias móveis representam uma significativa oportunidade para a educação, nesse contexto professores e alunos percebem o seu potencial no processo de ensino aprendizagem. Estudos na área investigam meios de combinar tecnologia e educação, através de softwares educacionais nos quais o usuário tem a possibilidade de acessar seu material de estudo incluindo e-mails e vídeo-aulas de maneira integrada em um ambiente online que permite aos professores e alunos um grande nível de interação (os AVAs) em qualquer lugar e a qualquer hora, isso por sua vez facilita a administração das tarefas diárias dos usuários com pouco tempo para estudar [Mühlbeier et. al. 2012] [Kaur \& Bhullar 2013].

Com relação às considerações dos autores conclui-se que tais tecnologias além de serem utilizadas para facilitar a vida, também podem ser exploradas para a promoção da educação. Neste cenário, as mesmas ganham espaço cada vez mais significativo sendo envolvidas em diversos contextos sociais. É notório o fato de que a necessidade tecnológica avança com rapidez e que ganha destaque sua enorme contribuição que vai desde pequenas tarefas diárias até momentos mais complexos tornando-os mais fáceis e rápidos [Mühlbeier et. al. 2012].

Esse espaço que a tecnologia vem ganhando na vida cotidiana da sociedade ganha destaque e inclusive é objeto de estudo de alguns pesquisadores. Estudos preliminares afirmam que $94 \%$ dos pesquisados confirmaram que o uso de aplicativos em dispositivos móveis potencializa a vida das pessoas no espaço urbano. Isso nos leva a concluir que a sociedade está vivendo um momento de evolução tecnológica e a cada interação e conexão as cidades se modificam e reescrevem sua história, proporcionando ao cidadão a possibilidade de participação efetiva e ampliada [Silva \& Urssi, 2015].

\section{Metodologia}

Este artigo apresenta o desenvolvimento de um aplicativo para dispositivos móveis, denominado EcoÁgua, que implementa o desenvolvimento da consciência do consumo hídrico responsável. Para idealizar o aplicativo, foi realizada uma pesquisa na loja virtual Play Store, disponível em dispositivos móveis para Android. Inicialmente foi verificado o que estava sendo produzido no Brasil quanto a aplicativos para dispositivos móveis voltados a sustentabilidade. Nessa busca constataram-se cinco aplicativos voltados para o uso da água, dos quais quatro nacionais e um internacional. 
O locus da pesquisa é uma Unidade Acadêmica de Educação a Distância de uma Universidade Pública brasileira. Esta unidade acadêmica atua na educação a distância (EAD) desde 2005 ofertando nove cursos de graduação com aulas presenciais distribuídas em diversos polos. A pesquisa tem como público alvo a comunidade acadêmica da unidade que é composta de servidores, colaboradores e alunos. Quanto ao público discente, o estudo trabalha com os alunos das graduações de dois dos polos presenciais do interior do estado de Pernambuco.

Parte das ações da pesquisa, como as que visam promover a informação, foram veiculadas por meio do ambiente virtual de aprendizagem. Tratando-se do EcoÁgua, será promovido o aprendizado de consumo consciente de água por meio de aplicativo para dispositivos móveis fazendo uso da aprendizagem móvel (m-learning), uma vez que o aplicativo traz telas de dicas de consumo consciente e permite que o usuário ponha em prática ações de consumo responsável.

\section{Trabalhos Relacionados}

Com base no levantamento feito na loja virtual Play Store, foram encontrados cinco aplicativos com características para incentivar o consumo consciente da água. São eles:

- Banho Rápido

- Nossa Água

- Dropcounter

- Pegada Hídrica

- Projeto Água Viva

Após a análise dos aplicativos, não foram encontrados aplicativos brasileiros que combinassem ao mesmo tempo as funcionalidades de calculadora de consumo de água em atividades diárias, dicas de uso consciente, ranking de usuários para comparar o gasto, acompanhamento do histórico de gasto hídrico e cálculo aproximado da média real de consumo, para que, por meio do comando de voz, o usuário possa iniciar, pausar e finalizar a contagem de uso da água sem interromper suas atividades ou ter que manusear o aparelho móvel para isso.

Com base nesses aplicativos encontrados, verifica-se a necessidade de preencher essas lacunas criando um aplicativo que una todas estas características com o objetivo de deixá-lo o mais próximo possível da realidade de consumo do usuário brasileiro. $\mathrm{O}$ usuário pode acompanhar o consumo diário, por meio do Ranking Ecoconsciente, comparar seu consumo com o consumo per capita, conforme dados de órgãos reguladores, do país que mais consome, do que menos consome e do Brasil para assim comparar com seu gasto e ter uma ideia se realmente economizou água.

\section{O Aplicativo}

O EcoÁgua está sendo desenvolvido para o sistema operacional Android, plataforma do Google mais utilizada em dispositivos móveis atualmente. Seu desenvolvimento foi 
V Congresso Brasileiro de Informática na Educação (CBIE 2016)

Anais dos Workshops do V Congresso Brasileiro de Informática na Educação (CBIE 2016)

iniciado usando a plataforma MIT App Inventor ${ }^{1}$ que torna viável a programação de aplicativos por meio de blocos.

Com essa ferramenta é possível desenvolver aplicativos personalizados e produzir de maneira rápida um protótipo, bem como testá-lo. Porém, foi verificado que era preciso agregar novas plataformas para sua programação devido às limitações do App Inventor para algumas funcionalidades, tais como: comando de voz; para iniciar, pausar e finalizar o uso da calculadora de consumo de água; bem como a organização desses dados de gasto hídrico em gráficos com histórico de consumo.

É importante saber que por trás de uma aplicação sendo executada explicitamente em algum aparelho, há diversas camadas de bibliotecas e funções rodando implicitamente nos aparelhos, ou seja, a plataforma Android trabalha com várias camadas em uma mesma aplicação, e isso causa uma complexidade elevada para desenvolver a aplicação.

Devido a sua estrutura complexa, para desenvolver aplicações na plataforma Android, é necessário à instalação de um ambiente de desenvolvimento. $\mathrm{O}$ primeiro software a ser instalado foi o Java JDK, que serve de base para todo desenvolvimento Android. Em seguida foi necessário fazer a instalação e configuração do Android Studio, que é um ambiente integrado de desenvolvimento oferecido pelo Google.

Por fim, foi preciso fazer a instalação de pacotes utilizando o Android SDK (Kit de Desenvolvimento de Software) Manager, responsável pelo gerenciamento dos pacotes e bibliotecas da plataforma. O SDK inclui documentação, códigos e utilitários. Toda linguagem utilizada no desenvolvimento da aplicação foi o JAVA.

Foi utilizado o ADP plug-in, que o sistema Android disponibiliza para a plataforma eclipse. $\mathrm{O}$ uso desta ferramenta pretende incentivar o consumo consciente de água por meio da visualização do gasto hídrico em atividades diárias, conforme observado na Figura 1 comparando ao consumo de outros usuários e ao mesmo tempo trazer dicas para a redução deste consumo.
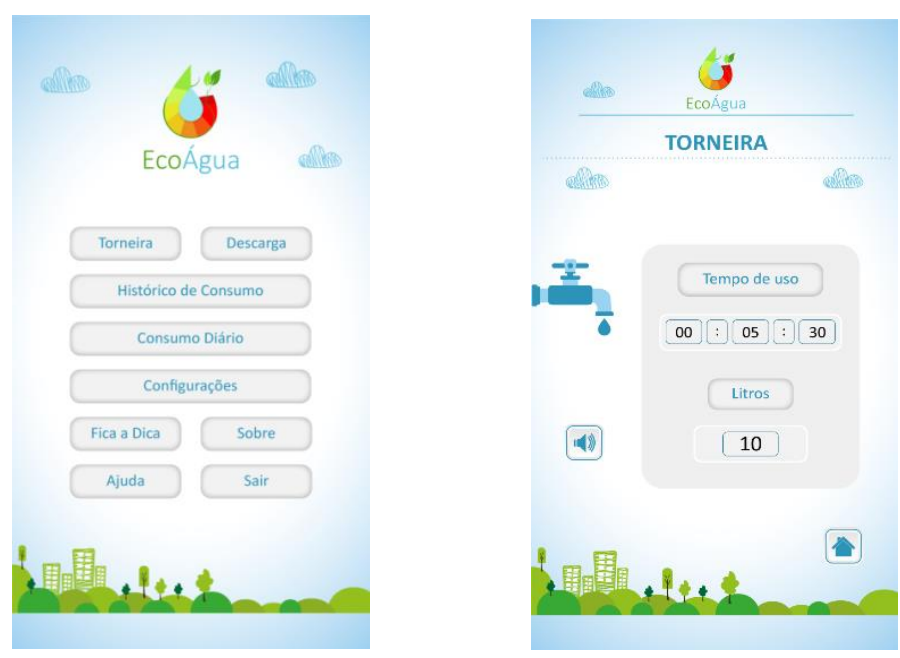

Figura 1. Telas do EcoÁgua

\footnotetext{
${ }^{1}$ MIT APP INVENTOR. Disponível em: <http://appinventor.mit.edu/explore/>
} 
V Congresso Brasileiro de Informática na Educação (CBIE 2016)

Anais dos Workshops do V Congresso Brasileiro de Informática na Educação (CBIE 2016)

Por meio da visualização do gasto hídrico diário juntamente com as dicas de redução de consumo, o usuário tem oportunidade de aprender de maneira simples sobre a importância do uso consciente da água, recurso natural indispensável para vida e já em escassez em diversas regiões. Tais funcionalidades podem ser observadas na Figura 2.
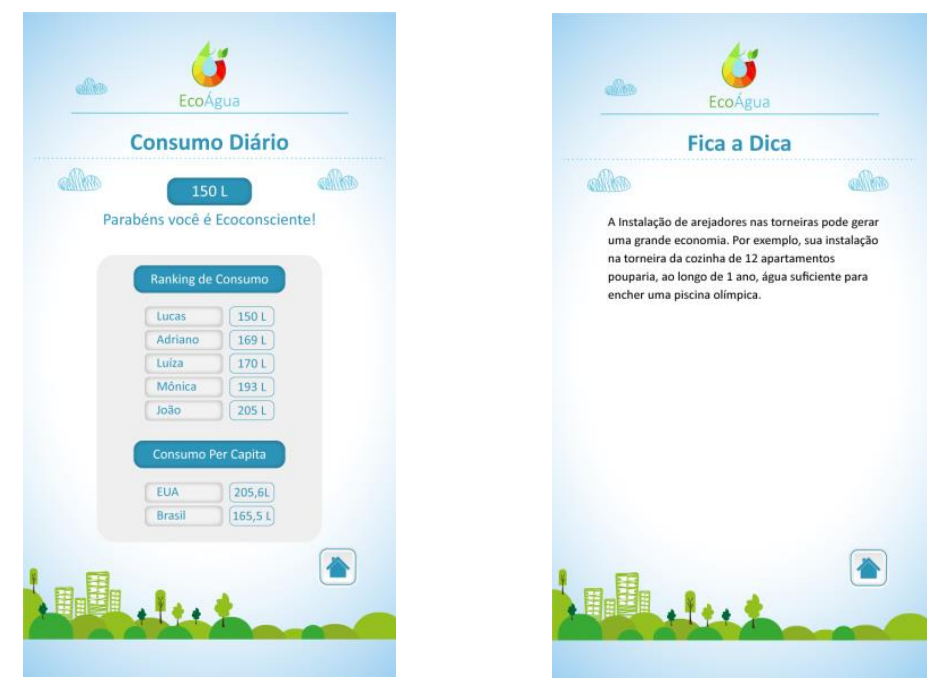

Figura 2. Consumo Diário e Dicas

Além de poder acompanhar o consumo diário, por meio do Ranking Ecoconsciente, o usuário poderá comparar seu consumo com o consumo per capita, conforme dados de órgãos reguladores, do país que mais consome, do que menos consome e do Brasil para assim comparar com seu gasto e ter uma ideia se realmente economizou água. É possível comparar com o histórico semanal, mensal e anual, conforme observado na Figura 3.
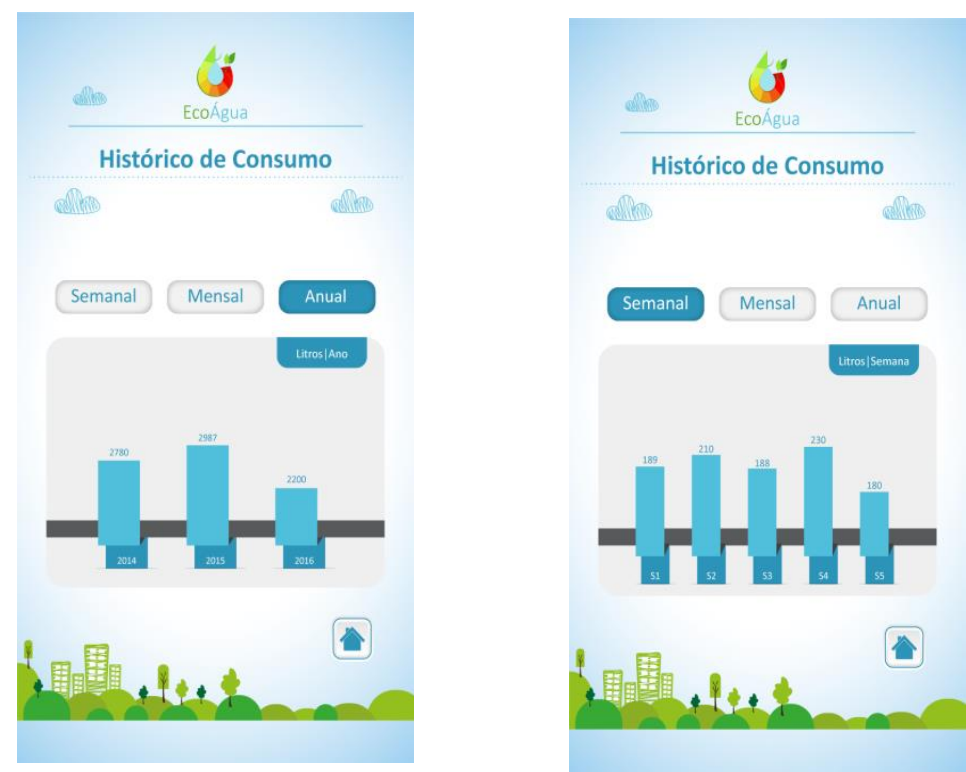

Figura 3. Consumo Semanal e Anual 
O aplicativo contabilizará o gasto em atividades diárias, para isso, utilizará uma calculadora e cronômetro que fará a contabilização em 1/s. O cronômetro será acionado por comando de voz tanto para iniciar, pausar como finalizar a contagem. Além disso, o EcoÁgua trará dicas de consumo consciente, um ranking de usuários para comparação do gasto hídrico e o histórico de consumo do usuário.

\section{Conclusão e Trabalhos Futuros}

Através do desenvolvimento do aplicativo e da pesquisa a ele associada, pretende-se promover a informação com o objetivo de influenciar nos primeiros passos para o processo de sensibilização e conscientização sustentável. Nesse sentido, contribuir com o desenvolvimento de um comportamento reflexivo dos sujeitos quanto ao seu papel frente às questões ambientais e na adoção da cultura de consumo consciente.

O uso do EcoÁgua, pretende incentivar o consumo consciente da água por parte dos envolvidos na pesquisa. Imagina-se que o usuário ao visualizar e comparar o seu gasto hídrico com o de outros usuários, bem como com a média de consumo brasileira e do país que mais consome tenha uma noção sobre seu consumo e/ou desperdício.

Com o auxílio de questionários que serão aplicados também na última fase de investigação, será possível verificar se houve alguma mudança na percepção sobre questões ambientais e sustentabilidade, já que isso foi investigado no primeiro questionário. A partir dos dados coletados no aplicativo, quanto ao uso por parte dos usuários, será possível verificar se houve uma evolução relacionada a redução de consumo de água dos usuários comparando seus respectivos dados ao longo do tempo de uso por meio de seu histórico de consumo.

Com essa temática pretende-se enfatizar a importância de uma maior participação das Universidades com relação ao desenvolvimento de ações que estimulem o desenvolvimento de uma consciência sustentável e contribuir com o incentivo a novas pesquisas que venham desenvolver trabalhos no intuito de inserir cada vez mais as instituições de nível superior neste cenário. Deseja-se destacar que o crescente uso das tecnologias não deve ser apenas observado como fator negativo em relação à sustentabilidade, mas considera-se que tais tecnologias podem ser usadas a favor do meio ambiente difundindo sobre aspectos ambientais e de sustentabilidade não só como meio de informação, mas com diversas aplicações, sobretudo fazendo uso das tecnologias móveis dentro do contexto da educação ambiental.

\section{Referências}

ARAÚJO, M. M. et al. Dropless App - Uso Sustentável da Água Através dos Smartphones. In: EXPOSIÇÃO DA PESQUISA EXPERIMENTAL EM COMUNICAÇÃO, 22., 2015, São Paulo. Anais eletrônicos...São Paulo: UnP, 2015. Disponível em: $<$ http://www.portalintercom.org.br/anais/nordeste2015/expocom/ EX47-2619-1.pdf >. Acesso em: 180 nov. 2015.

CARVALHO, A. P. et. al. Estudo da degradação ambiental do açude de Bodocongó em Campina Grande - PB. Revista de Engenharia Ambiental - Espírito Santo do Pinhal. v. 6, n. 2, p. 293-305, maio/ago. 2009. Disponível em: $<$ http://ferramentas.unipinhal.edu.br/engenhariaambiental/viewart icle.php?id=220\&layout=abstract $>$. Acesso em: 21 jul. 2015. 
V Congresso Brasileiro de Informática na Educação (CBIE 2016)

Anais dos Workshops do V Congresso Brasileiro de Informática na Educação (CBIE 2016)

DETERDING, S. Gamification: Designing for motivation. Interactions, v.19, n. 4, p. 14-17, jul./ago., 012. Disponível em: <http://dx.doi.org/10.1145/2212877.2212883>. Acesso em: 23 ago. 2015.

DIAS, G. F. Educação Ambiental: Princípios e Práticas. 8. Ed. São Paulo: Gaia, 2003. p. 74.

FRANÇA, R.M.; REATEGUI, E. B. SMILE-BR: Aplicação de conceitos de gamificação em um ambiente de aprendizagem baseado em questionamento. In: Simpósio Brasileiro de Informática na Educação - SBIE, 2., 2013, Porto Alegre. Anais Eletrônicos...Porto Alegre: 2013. Disponível em: $<$ http://www.br-ie.org/pub/index.php/sbie/article/view/2515/2173>. Acesso em 23 ago. 2015.

GIACOMIN, G.S.; OHNUMA, A. A. A pegada hídrica como subsídio a ações de educação ambiental. Ambiente e Educação. Revista de Educação Ambiental, Rio Grande, v. 17, n. 1, 2012. Disponível em: $<$ http://www.seer.furg.br/ambeduc/article/view/2433/1671>. Acesso em: 23 Jun. 2015.

GOULART, M.; CALLISTO, M. Bioindicadores de qualidade de água como ferramenta em estudos de impacto ambiental. Revista da FAPAM, ano 2, n. 1, 2003 Disponível em: $<$ http://www.santoangelo.uri.br/ briseidy/P\%F3s\%20Licenciamento\%20Ambiental/bioindicadores \%2019.10.2010.pdf $>$. Acesso em: 05 maio 2015.

KAUR, A.; BHULLAR, M. S. A New Method of Learning: MLearning (Mobile Learning). In: WORLD CONGRESS ON ENGINEERING AND COMPUTER SCIENCE, 1. 2013, San Francisco. Atas...San Francisco, 2013. p. 1-3. Disponível em: $<$ http://www.iaeng.org/publication/WCECS2013/WCECS2013_ pp187-189.pdf >. Acesso em: 23 fev. 2016.

MARCOMIN, F. E.; SILVA, A. D. V. A. A Sustentabilidade No Ensino Superior Brasileiro: Alguns Elementos a Partir da Prática de Educação Ambiental na Universidade. Revista Contrapontos, Itajaí, v. $09, \quad$ n. 2, p. 104-117, maio/ago. 2009. Disponível em: $<$ http://www6.univali.br/seer/index.php/rc/article/view/999> Acesso em: 03 maio 2015.

MÜHLBEIER, A. R. K. et al. eNIGMA e M-Learning: jogo educativo trabalhando o raciocínio lógico através de dispositivos móveis. Revista Brasileira de Computação Aplicada, Passo Fundo, v. 4, n. 2, p. 92-102, out. 2012. Disponível em: <http://www.upf.br/seer/index.php/rbca/article/view/2450/1879>. Acesso em: 27 jun. 2015.

O’MALLEY, C.; VAVOULA, G.; GLEW, J.P.;TAYLOR, J. SHAPLES, M. ; LEFRERE, P. MOBIlearn WP4 - Guidelines for Learning/Teaching/Tutoring in a Mobile Environment. 2003. Disponível em: $<$ https://hal.archives-ouvertes.fr/hal-00696244/document $>$. Acesso em 23 ago. 2016.

ORGANIZAÇÃO DAS NAÇÕES UNIDAS PARA A EDUCAÇÃO, A CIÊNCIA E A CULTURA (UNESCO), Relatório Mundial das Nações Unidas sobre Desenvolvimento dos Recursos Hídricos: Água para um mundo Sustentável, 2015. Disponível em: http://www.unesco.org/new/fileadmin/MULTIMEDIA/HQ/SC/images/WWDR2015ExecutiveSum mary_POR_web.pdf. Acesso em: 19 nov. 2015.

SILVA, R. J. S.; URSSI, N. J. UrbX - Como os aplicativos móveis potencializam a vida urbana. Revista de Iniciação Científica, Tecnológica e Artística, São Paulo, v. 5, n. 1, jun. 2015. Disponível em:

$<$ http://www1.sp.senac.br/hotsites/blogs/revistainiciacao/wpcontent/uploads/2015/06/98_artigo_Inic iacao_ed-vol5_n1_2015.pdf $>$. Acesso em: 26 jun. 2015.

TRAXLER, J. Current State of Mobile Learning. In Mobile Learning: Transforming the Delivery of Education and Training, AU Press, Athabasca University. cap. 1, p. 1-24. 2009.

VEIGA W., Campos, F., Braga, R., David, J. M. LUDOS: Uma Infraestrutura para Gamificação em Ecossistemas de E-learning. In: Simpósio Brasileiro de Informática na Educação - SBIE, 26., 2015, Porto Alegre. Anais..., Porto Alegre: 2015. Disponível em: <http://www.brie.org/pub/index.php/sbie/article/view/5289>. Acesso em: 23 ago. 2016. 